\title{
Erratum
}

\section{Entanglement invariants and phylogenetic branching}

\section{J.G. Sumner • P.D. Jarvis}

Published online: 11 July 2006

(C) Springer-Verlag 2006

J. Math. Biol. (2005)

\section{Digital Object Identifier (DOI) 10.1007/s00285-004-0309-z}

Equation (20) on page 31 of "Entanglement invariants and phylogenetic branching" is presented as

$$
\mathcal{T}(h)=\sum h_{a_{1} a_{2} a_{3}} h_{b_{1} b_{2} b_{3}} h_{c_{1} c_{2} c_{3}} h_{d_{1} d_{2} d_{3}} \epsilon_{a_{1} b_{2}} \epsilon_{b_{2} c_{1}} \epsilon_{c_{2} d_{1}} \epsilon_{d_{2} a_{2}} \epsilon_{b_{2} d_{3}} \epsilon_{a_{3} c_{3}} .
$$

This expression is incorrect and needs to be replaced with

$$
\mathcal{T}(h)=\sum h_{a_{1} a_{2} a_{3}} h_{b_{1} b_{2} b_{3}} h_{c_{1} c_{2} c_{3}} h_{d_{1} d_{2} d_{3}} \epsilon_{a_{1} b_{1}} \epsilon_{a_{2} b_{2}} \epsilon_{c_{1} d_{1}} \epsilon_{c_{2} d_{2}} \epsilon_{a_{3} d_{3}} \epsilon_{b_{3} c_{3}} .
$$

The online version of the original article can be found at http://dx.doi.org/10.1007/s00285-004-0309-z.

J.G. Sumner $(\varangle)$ P P.D. Jarvis

School of Mathematics and Physics, University of Tasmania GPO,

Box 252-21, Hobart Tas 7001, Australia

e-mail: jsumner@utas.edu.au 